\title{
Visualization of mental disorders by molecular connectivity with omics platform
}

\author{
Elizabeth Heroux, Aminata Musa, Jae-Hyeon Cho, Diane E Heck and Hong-Duck Kim* \\ ${ }^{1}$ Department of Public Health, Division of Environmental Health Science, New York Medical College, USA \\ ${ }^{2}$ Institute of Animal Medicine, College of Veterinary Medicine, Gyeongsang National University, Korea
}

\begin{abstract}
Emerging evidence indicate various environmental factors include stressors cause mental illness. Currently, unmet medical outcomes and surveillance platform demand in the field of mental disorders to implement of sensitivity associated along with key biomarker in the process of detection, evaluation and validation. The use of omics, based on macromolecule dependent data analysis such as bioinformatics in mental disorders and in a health care assessment platform, has the potential to increase identification of risk genes and treatment option by validate functional efficacy of drug or recovery status of brain function utilize behavioral assessment and metabolomics include neuro-pharmacogenomics study. This concept sounds futuristic but ideal. If the multiple data bases around the world continue to grow, making identification of a strand more likely, then health professional include clinicians and lab scientists could predict the likelihood of a disease outbreak, drug addiction, and the probability of synaptic malfunction that a treatment will work. Being able to stratification of risk gene profile and biomarker monitoring prior to disease dissemination has the potential to greatly diminish the current health care economic burden. However, the implementation in the clinical setting may take time; the therapeutic alternative includes biological and synthetic product will be worth the wait. Herein, we conducted short review of mental illness which predominantly occurred and unknown causes by visualize identifying risk genes and functional susceptibility in the molecular diagnostic advances using Omics platform. In the future, it is likely that a majority of our treatment and care for patients or personal medicine/population medicine will be based on an omics strategy, molecular based risk assessment tools, as a safe and empower sensitivity to validate potential risk gene or therapeutic gene by adopting early detection risk management such as Integrated Omics.
\end{abstract}

\section{Introduction}

Mental health disorders are common and may have symptoms that are relatively mild for the individual or can range into severe. In 2010 it was estimated that the average disability-adjusted life years for mental and substance abuse disorders was 183.9 million. This accounted for $7.4 \%$ worldwide [1]. Mental health and substance abuse disorders grew a rapid $37 \%$ in 20 years due to an increase in population, aging, and increase in mental health care. The most common disorders in the general population are generalized anxiety disorder, major depressive disorder, eating disorders, pervasive developmental disorders, alcohol and substance abuse or dependence disorders, bipolar disorder and schizophrenia. The World Health Organization [2] defines risk assessment as "a systematic process for gathering, assessing and documenting information to assign a level of risk. It provides the basis for taking action to manage and reduce the negative consequences of acute public health risks."

Omics technologies include bioinformatics and data analyses are methodologies related to DNA, RNA, proteins and metabolites $[3,4]$. There are multiple different omics methods used in assessing drug, neuronal circuit using single cell genomics in mental pathogenesis of neuropsychiatric diseases [5-8]. The selected omics for examples, Genomics, Transcriptomics, Proteomics, Metabolomics, pharmacogenomics, Epigenetics, and neuro-toxicogenomics are widely applicable to predict risk gene to explicit pathogenesis of mental disorders. Additionally, mental health and substance abuse disorders were directly linked for 232,000 lives lost in 2010. At this time the most common ways to treat mental health and substance disorders is through molecular diagnostic, tool for therapeutic evaluation, and medication management if needed. Many mental health disorders are genetically linked. Researchers in clinic and pharmaceutics are interested in finding a way to identify individuals who have the biomarker for a mental health disorder. This can increase treatment effectiveness and options since right now patients may need to trail multiple different medications before finding the one that works best for them.

\section{Methods}

We conducted a literary search in Pubmed. A search key words consisted of "Depression and Biomarkers", "Mental Health AND global burden of disease" and "biomarkers AND mental health AND substance abuse." It has limited to articles which have been peer reviewed and are from 2013 or more recent. We focused on depression, schizophrenia and substance abuse, alcohol addiction, and PTSD since they are the rated disorders that are either common or can be extremely disabling, which has been demand due to lack of surveillance gene which we need to implement following concentrated efforts on validated value of biomarkers exploration with molecular based assessment tools in Omics platform.

Correspondence to: Hong Duck Kim, Department of Public Health, Division of Environmental Health Science, New York Medical College, Valhalla NY 10595, USA, Tel. +1-914-594-4259, Fax. +1-914-594-4576, E-mail. hongduck_kim@nymc.edu

Key words: mental disorders, depression, schizophrenia, alcohol use disorder, PTSD and personal medicine, molecular diagnostic, omics platform

Received: April 07, 2018; Accepted: April 17, 2018; Published: April 20, 2018 


\section{Results}

\section{Depression}

Depression is one of the most common mental health disorders. The National Institute of Mental Health states that there are approximately 16.1 million adults who have suffered from a depressive episode. A depressive episode is categorized as a two week time frame where there is a depressed mood, loss of interest, increase in guilt, and a change in sleeping patterns, eating patterns, and concentration. Not all individuals are going to develop all of these symptoms and the symptoms that are developed may be mild or severe with thoughts of wanting to end their own life.

A biomarker identified with depression is the inflammatory biomarker C-Reactive Protein (CRP). Individuals who have high levels of CRP are less likely respond to antidepressants in the serotonin reuptake inhibitor and the norepinephrine reuptake inhibitor category. The identification and use of this biomarker can assist in creating a differential on why a patient may not be responding to treatment [9]. A common way to proactively treat inflammation is to prescribe fish oil pills due to their high levels of Omega-3 Fatty Acids, which are known to reduce inflammation [10]. This biomarker is easy to obtain through a routine non-fasting lab which may increase its use in the clinical setting.

\section{Schizophrenia}

Schizophrenia is known to be genetic and similar to alcohol dependence. It is a gene by environment disease. Gene by environment means that the disorder begins to be symptomatic due to being triggered by something. The environmental trigger for schizophrenia is potentially multifactorial and inconclusive. Some studies have linked bullying or abuse to $35 \%$ of cases, maternal vitamin deficiency linked to $44 \%$ of cases, urban birth and upbringing to $35 \%$ of cases, and cannabis use being linked to $14 \%$ of adolescent cases [9]. Whereas the environmental trigger for alcohol disorders is of course alcohol. There have been multiple studies to try and determine potential biomarkers for schizophrenia, but many are inconclusive. Seven MicroRNA's were originally thought to be potential biomarker's for schizophrenia and showed potential based off of a lab test with a mouse subject. The mouse underwent a treatment for schizophrenia and show increase in microRNA changes. The theory was the range of microRNA changes could predict the effectiveness of the medication treatment. However, in human subjects the results were not repeated $[11,12]$.

Two other potential biomarkers were identified with potential to identify acute schizophrenia and the trait for schizophrenia but had a relatively low sample size which reduces its clinical significance. Nonetheless, startle habituation and Prepulse Inhibition (PPI) were both found to be elevated in cases of acute schizophrenia which needed hospitalization for stabilization. After treatment and hospitalization for an average of three months a majority of the individuals had a reduction in startle habituation. The PPI were elevated for patients at hospitalization and at discharge which speaks to the likeliness that the PPI may serve as a biomarker for the disease [13]. If the biomarker for schizophrenia can be confidently determined, then patient's carrying the schizophrenia gene can be identified before the onset of symptoms. Symptoms of schizophrenia may be hard to fully identify without the biomarker since patients suffering from schizophrenia may be paranoid, have auditory or visual hallucinations, and tend to isolate themselves.

\section{Alcohol use disorder}

Alcohol disorder is a dangerous disease that causes approximately 2.5 million deaths around the world a year. Several preliminary biomarkers have been developed to identify individuals who do not self-identify they are having trouble controlling their drinking. Two of these biomarkers are the Alanine Transaminase (ALT) and Aspartate Transaminase (AST) levels that are obtained during a routine lab test. However, there are some limitations to these tests since some heavy drinkers may genetically be designed for their body to handle larger quantities of alcohol. As risk gene in the etiology of alcohol dependence, it was reported dopamine transporter (DAT, gene symbol: SLC6A3) gene could be engaged in susceptibility in alcohol dependence. However, it still remains to be verify using further Omics tools such as GWAS and pharmacogenomics [16].

In addition, alcohol addiction causes breast cancer as well as neuronal inflammation. Using proteomics combined with mass spectrometric analysis as part of metabolomics approach, neuronal dysfunction include neuronal inflammation was examined in microglia cell following by applying stable isotope labelling [14, 15]. Today, it is highly demanded to explore biomarkers which we need to prevent young adults from alcohol addiction which has not available molecular diagnostic option in a clinical setting. This means that clinicians are only able to catch an alcohol use disorder after side effects from the heavy drinking have already taken place. In comparison the hundreds of subjects used in the clinical study to locate the biomarker cytosinephosphate-guanine now know they have a high probability to develop an alcohol use disorder if they activate their gene by consuming alcohol frequently.

\section{Post-Traumatic Stress Disorder (PTSD)}

PTSD is defined as "a disorder that develops in some people who have experienced a shocking, scary, or dangerous event." Although the "fight or flight" response is a natural reaction meant to be protective during a traumatic event, a person may be diagnosed as having PTSD if they continue to have reactions to the past event when they are no longer in any danger [17]. This is a mental health issue that affects victims of trauma and violence, and soldiers and survivors of war. With the aging population of soldiers and survivors of past wars, such as the war in Vietnam, Iraq War, and the war in Afghanistan, PTSD has been and will continue to be an ongoing problem. PTSD is one of many mental illnesses which are becoming more prevalent. With PTSD, there are molecular mechanisms as well as environmental factors which may be responsible for the onset of this disorder. The World Health Organization [2] defines risk assessment as "a systematic process for gathering, assessing and documenting information to assign a level of risk. It provides the basis for taking action to manage and reduce the negative consequences of acute public health risks." The following are steps taken to assess the risk of mental illness or to develop a risk assessment for a PTSD mental health outbreak: Hazard assessment, Exposure assessment, Context assessment.

Upon PTSD diagnosis pattern, it is influenced by mutations within genes or has been influenced by epigenetic causes. As a Potential mechanism, methylation be proposed a molecular alteration process that affects the activity of a large percentage of genes. It was indicated that epigenetic modification by methylation could influence on the development circuit of stress regulation in early life $[18,19]$. Moreover, as a susceptibility gene to PTSD have been implicated, for example, hypo-cortisolism or low cortisol levels may predispose individuals to PTSD as it may be a risk factor for maladaptive stress responses it could consider potential therapeutic option in that hydrocortisone may be used to prevent and treat PTSD [20]. Genetic polymorphisms in the FKBP5 gene, glucocorticoid receptor co chaperone protein, 
demonstrated genetically determined features of DA transmission lead to the development of PTSD in the correlation study of non-psychiatric patients between abuse and trauma. Interestingly, polymorphism of the DA transporter gene with an excess of the SLC6A39 repeat allele was found in PTSD patients. In case of Childhood abuse and adulthood trauma, each predicted PTSD symptoms and FKBP5 polymorphisms significantly interacted. The FKBP5 genotype was further linked to enhanced glucocorticoid receptor sensitivity, as reflected by dexamethasone hyper suppression, a hallmark feature of PTSD.

Similarly, one gene locus associated with PTSD, MAN2C1, provided evidence of a significant interaction with number of potentially traumatic events in predicting risk of PTSD. They concluded that PTSD is associated with dysregulation in immune system related genes in peripheral blood. The study suggests MAN2C1 could be associated in developing PTSD with respect to epigenetic variation, in particular, methylation level can moderate risk for PTSD risk with featured symptoms in trauma. Using animal model, it was further investigated that methylation changes in brain tissue following stress exposure and identified loci (e.g. Dlgap2) which expressed changes in both methylation and gene expression patterns in postmortem hippocampal tissue which are associated with PTSD [21].

In previous, study conducted the correlation between vulnerability to addiction in which the animal index locomotor responses to novelty can be used to examine the effects of trauma because it is looks at anxiety which has been shown to represent a predictive factor for the addictive properties of drug or animal vulnerability to drug [22]. To distinguish differences in locomotor response, rats were categorized as High Responders (HR) or low responders (LR). They found HR to be more vulnerable than LR rats by increased stress-evoked dopamine release in the nucleus of accumbens. Interestingly, Dopamine receptor function has been found to influence novelty-seeking behavior, therefore, the dopamine receptor antagonist haloperidol and the selective dopamine D1 receptor antagonist SCH23390 block novelty preference [22].

Among autosomal genes, gene to gene interaction between SLC6A3 and NR4A2 SNPs, which nicotinic acetylcholine receptors and genes involved in dopaminergic function, was shown highly significant which reflect nicotine dependence underlying mechanism likely NR4A2 Protein Product (NURR1) could regulates SLC6A3 transcription [23]. Similarly, Omics study conducted to examine if Single Nucleotide Polymorphism (SNP) in the gene coding for the alpha 4 subunit of the nicotinic acetylcholine receptor (CHRNA4) and DRD2 genotypes modulate the effect of nicotine on visuospatial attention and distractor interference. The study outcome represented that DRD2 but not CHRNA4 genotype modulated the effects of nicotine on distractor interference with DRD2 CC carriers. It suggests that individual variability in cognitive and neural effects of nicotine could be affected by both cholinergic and dopaminergic genetic variations [24].

By applying similar strategy utilizes Omics platform, it need to determine if difference genetic variance between cholinergic and dopaminergic may pose to the occurrence of substance abuse and PTSD in patient's samples.

Advanced assessment tools like Omics could improve early detection sensitivity and positioning of gene abnormality equipped with genome-wide association support include GWAS, RNA-sequences. It may provide strong evidence for genetic determinants of gene alteration which may involve in process of addiction and vulnerability strata in mental illness or disorders.
All serious mental health disorders, which include major depressive disorder, schizophrenia, and alcohol use disorder are multifactorial and have a multitude of environmental factors that likely contribute to the development of the disorder. Examples of these triggers are cannabis, maternal stress, in utero exposure to infection, malnutrition, traumatic events and bullying [25,26]. Currently the United States is experiencing a mental health outbreak with the ongoing opioid crisis. What is not immediately known to the public is why so many people begin using illicit drugs or abusing prescription medication.

In the event of a mental health outbreak scenario, it is possible to use biomarkers to identify the potential risk an individual has of developing a disorder. One way that this could be useful is screening military members for their genetic predisposition of developing PTSD, Depression, or Psychotic disorders. However, there is an ethical limitation to using an individual's medical risk for a disease as a job disqualifier that mental health community will have to ask themselves at some point in the future.

\section{Conclusions/ Future perspectives}

In present, there is not a federally approved clinical method of using biomarkers to treat mental health disorders. There have been multiple challenges that arose from the attempt to bring in clinical biomarkers into the clinical setting. These challenges are fraud, clinical setting limitations, and personal biases. Even with these challenges, many for profit companies have started using preliminary biomarker results in patient care. In 2015 in Colorado Springs, every single one of our patients that that we send to an inpatient facility received an oral swab and was removed off of all medications they came in to the facility with and was placed on the medication that was supposedly best for them based off of their genetics. Ideally, more concreate biomarkers will be identified which allow clinician's to easily identify the best pharmaceutical option for a patient prior to multiple trials. The average antidepressant makes take 2-4 weeks to reach therapeutic dose and every medication does not work the same for each person, which delays a patient's treatment while they try multiple drugs for 2-4 weeks.

Reflections in advance reports, one of Omics application such a microarray analysis, and metabolomic which can use to treat trauma related disorders and substance abuse. To further determine unveil molecule program circuit as disease determinants with functional identification with relevant molecular processes, integrated Omics tools equipped imaging methodology could be considered to visualize the functional impairment and structural alteration which genes are responsible for a disorder by looking at global gene expression profiles in tissues using animal model or patient's samples. In addition, improvement of personal care system with facilitate communication utilize electronical medical record with regard to susceptible or mutation prone- the genes we can create effective treatments, and even design medication specific to an individual's experience with the disorder. Overall, the future perspective of using Omics platform shed light on venue in biomarker exploration, innovation and evaluation of assessment procedure in a clinical setting for the treatment of mental health disorder looks promising.

\section{References}

1. Whiteford HA, Degenhardt L, Rehm J, Baxter AJ, Ferrari AJ, et al. (2013) Global burden of disease attributable to mental and substance use disorders: findings from the Global Burden of Disease Study 2010. Lancet 382: 1575-1586. [Crossref]

2. World Health Organization (2012) Rapid Risk Assessment of Acute Public Health Events. 
3. Iourov IY, Vorsanova SG, Yurov YB (2012) Single cell genomics of the brain: focus on neuronal diversity and neuropsychiatric diseases. Curr Genomics 13: 477-488. [Crossref]

4. Bergholz TM, Moreno Switt AI, Wiedmann M (2014) Omics approaches in food safety: fulfilling the promise? Trends Microbiol 22: 275-281. [Crossref]

5. Fink JK, Heiman-Patterson T, Bird T, Cambi F, Dubé MP, et al. (1996) Hereditary spastic paraplegia: advances in genetic research. Hereditary Spastic Paraplegia Working group. Neurology 46: 1507-1514. [Crossref]

6. Lipska BK, Peters T, Hyde TM, Halim N, Horowitz C, et al. (2006) Expression of DISC1 binding partners is reduced in schizophrenia and associated with DISC1 SNPs. Hum Mol Genet 15:1245-1258. [Crossref]

7. Davies W (2013) Using mouse models to investigate sex-linked genetic effects on brain, behaviour and vulnerability to neuropsychiatric disorders. Brain Res Bull 92: 12-20. [Crossref]

8. Iwase S, Berube NG, Zhou Z, Kasri NN, Battaglioli E, et al. (2017) Epigenetic Etiology of Intellectual Disability. J Neurosci 37: 10773-10782. [Crossref]

9. Uher R (2014) Gene-environment interactions in severe mental illness. Front Psychiatry 5: 48. [Crossref]

10. Ellulu MS, Khaza'ai H,Abed Y, Rahmat A, Ismail P, et al. (2015) Role of fish oil in human health and possible mechanism to reduce the inflammation. Inflammopharmacology 23: 79-89. [Crossref]

11. Lai CY, Lee SY, Scarr E, Yu YH, Lin YT, et al. (2016) Aberrant expression of microRNAs as biomarker for schizophrenia: from acute state to partial remission, and from peripheral blood to cortical tissue. Transl Psychiatry 6: e717. [Crossref]

12. Beveridge NJ, Gardiner E, Carroll AP, Tooney PA, Cairns MJ (2010) Schizophrenia is associated with an increase in cortical microRNA biogenesis. Mol Psychiatry 15: 1176-1189. [Crossref]

13. Mena A, Ruiz-Salas JC, Puentes A, Dorado I, Ruiz-Veguilla M, et al. (2016) Reduced Prepulse Inhibition as a Biomarker of Schizophrenia. Front Behav Neurosci 10: 202. [Crossref]

14. Goshe MB, Smith RD (2003) Stable isotope-coded proteomic mass spectrometry. Curr Opin Biotechnol 14: 101-109. [Crossref]
15. Liu B, Barber DS, Stevens SM Jr (2012) Stable isotope labeling with amino acids in cell culture-based proteomic analysis of ethanol-induced protein expression profiles in microglia. Methods Mol Biol 829: 551-565. [Crossref]

16. van der Zwaluw CS, Engels RC, Buitelaar J, Verkes RJ, Franke B, et al. (2009) Polymorphisms in the dopamine transporter gene (SLC6A3/DAT1) and alcoho dependence in humans: a systematic review. Pharmacogenomics 10: 853-866. [Crossref]

17. National Institute of Mental Health (2016) Post-Traumatic Stress Disorder.

18. Roth TL, Zoladz PR, Sweatt JD, Diamond DM (2011) Epigenetic modification of hippocampal Bdnf DNA in adult rats in an animal model of post-traumatic stress disorder. J Psychiatr Res 45: 919-926. [Crossref]

19. Perroud N, Paoloni-Giacobino A, Prada P, Olié E, Salzmann A, et al. (2011) Increased methylation of glucocorticoid receptor gene (NR3C1) in adults with a history of childhood maltreatment: a link with the severity and type of trauma. Transl Psychiatry 1: e59. [Crossref]

20. Sherin JE, Nemeroff CB. (2011) Post-traumatic stress disorder: the neurobiologica impact of psychological trauma. Dialogues Clin Neurosci 13: 263-278. [Crossref]

21. Uddin M, Galea S, Chang SC, Aiello AE, Wildman DE, et al. (2011) Gene expression and methylation signatures of MAN2C1 are associated with PTSD. Dis Markers 30: 111-121. [Crossref]

22. Belin D, Deroche-Gamonet V (2012) Responses to novelty and vulnerability to cocaine addiction: contribution of a multi-symptomatic animal model. Cold Spring Harb Perspect Med 2. [Crossref]

23. Bergen AW, Conti DV, Van Den Berg D, Lee W, Liu J, et al. (2009) Dopamine genes and nicotine dependence in treatment-seeking and community smokers. Neuropsychopharmacology 34: 2252-2264. [Crossref]

24. Ahrens S, Markett S, Breckel TP, Behler O, Reuter M, et al. (2015) Modulation of nicotine effects on selective attention by DRD2 and CHRNA4 gene polymorphisms. Psychopharmacology (Berl) 232: 2323-2331. [Crossref]

25. Uher R, Tansey KE, Dew T, Maier W, Mors O, et al. (2014) An inflammatory biomarker as a differential predictor of outcome of depression treatment with escitalopram and nortriptyline. Am J Psychiatry 171: 1278-1286. [Crossref]

26. Chan MK, Cooper JD, Bahn S (2015) Commercialisation of Biomarker Tests for Mental Illnesses: Advances and Obstacles. Trends Biotechnol 33: 712-723. [Crossref]

Copyright: (C)2018 Heroux E. This is an open-access article distributed under the terms of the Creative Commons Attribution License, which permits unrestricted use, distribution, and reproduction in any medium, provided the original author and source are credited. 